\title{
Validez de la prueba de actividad enzimática de la glucocerebrosidasa para el diagnóstico de enfermedad de Gaucher, revisión sistemática
}

\author{
Validity of the Enzymatic Activity Test of Glucocerebrosidase for the \\ Diagnosis of Gaucher Disease, a Systematic Review
}

\section{Validade do teste de atividade enzimática da glucocerebrosidase para o diagnóstico da doença de Gaucher, revisão sistemática}

\author{
Lina María Vera-Cala, MD., MSc., PhD. * \\ Sergio Eduardo Serrano-Gómez, MD., MSc. ** \\ Alexandra Cortés, Econ., PhD. *** \\ Ismael Estrada, Econ., MSc. $* * * *$ \\ Aurora Gáfaro, L. Mat., PhD. *****
}

\section{Resumen}

Introducción: La enfermedad de Gaucher es un trastorno metabólico por deficiencia o ausencia de enzima $\beta$ Glucosidasa Ácida. El diagnóstico se sospecha clínicamente, pero requiere confirmación mediante medición, en leucocitos (estándar de oro) o en sangre seca sobre papel de filtro, de la actividad de la enzima $\beta$-Glucosidasa Ácida. Objetivo: Evaluar la validez de la medición de la actividad de la enzima $\beta$-Glucosidasa Ácida en sangre seca en papel de filtro, comparada con el estándar de oro, para el diagnóstico de enfermedad de Gaucher en pacientes con sospecha clínica.
Metodología: Se hizo una revisión sistemática de literatura. Se construyó y validó una pregunta PICO. Se usó una estrategia de búsqueda genérica con base en los términos clave (Gaucher Disease y Dried Blood Spot Analysis). Dos evaluadores independientes revisaron, evaluaron la calidad y extrajeron la información de los artículos. Resultados: Descartando los duplicados, se obtuvieron 47 artículos. Se evaluaron los textos completos de cuatro, y tres de ellos fueron excluidos al aplicar criterios de inclusión y exclusión. El artículo incluido tuvo una calidad excelente y mostró que la actividad enzimática de la glucocerebrosidasa en sangre seca tuvo sensibilidad de $82.3 \%$ y especificidad de $94.0 \%$ con un punto de corte de $0.0-2.75$ y sensibilidad de $88.2 \%$ y

* Médico y Cirujano, magíster en Epidemiología, doctorado de Epidemiología, grupo de Investigación en Demografía, Salud Pública y Sistemas de Salud, Facultad de Salud, Universidad Industrial de Santander, Bucaramanga, Colombia.

** Médico y Cirujano, magíster en Epidemiología, grupo de Investigación en Demografía, Salud Pública y Sistemas de Salud, Facultad de Salud, Universidad Industrial de Santander, Bucaramanga, Colombia.

*** Economista, doctorado en Economía, grupo de Investigación Estudios en Microeconomía Aplicada y Regulación, Facultad de Ciencias Humanas, Universidad Industrial de Santander, Bucaramanga, Colombia

**** Economista, magíster en Economía, grupo de Investigación Estudios en Microeconomía Aplicada y Regulación, Facultad de Ciencias Humanas, Universidad Industrial de Santander, Bucaramanga, Colombia.

***** Licenciatura en Matemáticas y Computación, doctorado en MatemáticaAplicada, grupo de Investigación en Demografía, Salud Pública y Sistemas de Salud, Facultad de Salud, Universidad Industrial de Santander, Bucaramanga, Colombia. 
especificidad de $88.5 \%$ con un punto de corte de $0.0-4.4$ Conclusiones: La medición enzimática de la $\beta$-glucosidasa ácida en sangre seca es una excelente prueba para diagnóstico inicial de enfermedad de Gaucher. Sin embargo, no es una prueba concluyente. [Vera-Cala LM, SerranoGómez SE, Córtes A, Estrada I, Gáfaro A. Validez de la prueba de actividad enzimática de la glucocerebrosidasa para el diagnóstico de enfermedad de Gaucher, revisión sistemática. MedUNAB 2017; 20(2): 201-206].

Palabras Claves: Enfermedad de Gaucher; Pruebas con Sangre Seca; Leucocitos; Sensibilidad y Especificidad; Diagnóstico; Glucosilceramidasa.

\section{Abstract}

Introduction: The Gaucher disease is a metabolic disorder due to deficiency or absence of acid $\beta$-glucosidase enzyme. The diagnosis is clinically suspected, but requires confirmation by measuring the activity of acid $\beta$-glucosidase enzyme in leukocytes (gold standard) or in dried blood on filter paper. Objective: To assess the validity of the measurement of acid $\beta$-glucosidase enzyme activity in dried blood on filter paper compared to the standard of gold, for the diagnosis of Gaucher disease in patients with clinical suspicion. Methodology: A systematic review of literature was carried out. A PICO question was constructed and validated. A generic search strategy was used based on the key terms (Gaucher Disease and Dried Blood Spot Analysis). Two independent evaluators reviewed and assessed the quality, and extracted information from the articles. Results: Discarding the duplicates, 47 articles were obtained. Four complete texts were evaluated, and three of them were excluded when applying inclusion and exclusion criteria. The article that was included had an excellent quality and showed that the enzymatic activity of glucocerebrosidase in dried blood had a sensitivity of $82.3 \%$, a specificity of $94.0 \%$ with a cut-off of $0.0-2.75$, a sensitivity of $88.2 \%$ and specificity of $88.5 \%$ with a cut-off point of $0.0-4.4$. Conclusions: The enzymatic measurement of acid $\beta$ glucosidase in dried blood is an excellent test for an initial diagnosis of Gaucher disease; however, it is not a conclusive proof. IVera-Cala LM, Serrano-Gómez SE, Córtes A, Estrada I, Gáfaro A. Validity of the Enzymatic Activity Test of

\section{Introducción}

La enfermedad de Gaucher (EG) es un trastorno metabólico de depósito lisosomal autosómico recesivo ocasionado por la deficiencia o ausencia de la enzima $\beta$-Glucosidasa ácida (o Glucocerebrosidasa) (1). Esta deficiencia enzimática favorece la acumulación del sustrato glucocerebrósido en los lisosomas de macrófagos y monocitos que conducen a la hipertrofia del sistema lisosomal celular que infiltra el tejido del cerebro, los pulmones, la medula ósea, el bazo y el hígado principalmente, lo que genera daño celular y disfunción orgánica $(2,3)$. La EG tiene una prevalencia mundial de $1 / 40,000$ a 1/60,000 nacidos vivos (4), en el país no existen estudios que determinen la prevalencia de EG en población general. Uribe y colaboradores encontraron una prevalencia de $2.15 \%$ de EG en pacientes con sospecha clínica(5).
Glucocerebrosidase for the Diagnosis of Gaucher Disease, a Systematic Review. MedUNAB 2017; 20(2): 201-206].

Keywords: Gaucher Disease; Dried Blood Spot Testing; Leukocytes; Sensitivity and Specificity; Diagnosis; Glucosylceramidase.

\section{Resumo}

Introdução: A doença de Gaucher é um transtorno metabólico devido a deficiência ou ausência de enzima de $\beta$-Glucosidase. O diagnóstico é clinicamente suspeitado, mas requer confirmação medindo, em leucócitos (padrão-ouro) ou em sangue seco em papel de filtro, a atividade de enzima de $\beta$ Glucosidase. Objetivo: Avaliar a validade da medida da atividade de enzima de $\beta$-Glucosidase em sangue seco em papel de filtro, em comparação com o padrão-ouro, para o diagnóstico de doença de Gaucher em pacientes com suspeita clínica. Metodologia: Foi feita uma revisão sistemática da literatura. Uma questão PICO foi construída e validada. Uma estratégia de pesquisa genérica foi utilizada com base nos títulos-chave (Doença de Gaucher e Análise de Pontos de Sangue Seco). Avaliaram dois avaliadores independentes, avaliaram a qualidade e extraíram informações dos artigos. Resultados: Descartando as duplicatas, foram obtidos 47 artigos. Os textos completos de quatro foram avaliados e três deles foram excluídos ao aplicar critérios de inclusão e exclusão. $\mathrm{O}$ artigo incluído teve uma excelente qualidade e mostrou que a atividade enzimática da glucocerebrosidase em sangue seco teve sensibilidade de $82.3 \%$ e especificidade de $94.0 \%$ com um corte de $0.0-2.75$ e sensibilidade de $88.2 \%$ e especificidade de $88.5 \%$ com ponto de corte de 0.0-4.4. Conclusões: A medida enzimática da $\beta$-glucosidase ácida em sangue seco é um excelente teste para o diagnóstico inicial de doença de Gaucher. No entanto, não é uma prova conclusiva. [Vera-Cala LM, Serrano-Gómez SE, Córtes A, Estrada I, Gáfaro $A$. Validade do teste de atividade enzimática da glucocerebrosidase para o diagnóstico da doença de Gaucher, revisão sistemática. MedUNAB 2017; 20(2): 201-206].

Palavras-chave: Doença de Gaucher; Teste em Amostras de Sangue Seco; Leucócitos; Sensibilidade e Especificidade; Diagnóstico; Glucosilceramidase.

La EG se clasifica en tres formas clínicas, basándose en la ausencia (tipo I) o presencia (tipos II y III) de afección neurológica, sin embargo, cada vez se encuentra mayor heterogeneidad fenotípica en estos grupos y hay reportes de fenotipos intermedios entre los tipos II y III. La presentación más frecuente es la tipo I, que constituye alrededor del 90\% de los casos reportados (4). La EG tipo I se puede diagnosticar a cualquier edad, la progresión puede ser lenta o rápida y el grado de afectación visceral moderado a severo, por lo general causa hepato-esplenomegalia, pancitopenia, dolor óseo, y fracturas, pero no tiene compromiso neurológico. El tipo II de la enfermedad se presenta en recién nacidos y causa daño cerebral grave por lo que la mayoría de los pacientes fallecen antes de los 2 años de edad. El tipo III suele debutar en la infancia o en la adolescencia y puede cursar con afectación visceral (hepatoesplenomegalia) además de afectación neurológica 
progresiva, que dependiendo de su velocidad de evolución puede llegar a ser mortal(6).

Realizar el diagnóstico precoz de la EG es importante debido a que, en general, los daños que produce la enfermedad son irreversibles(7). El diagnóstico de la EG se sospecha basándose en los datos clínicos, pero requiere confirmación mediante pruebas de laboratorio que midan la actividad de la $\beta$-Glucosidasa ácida (3), la cual puede ser medida en leucocitos (el actual estándar de oro) o en sangre seca sobre papel de filtro (8). En la actualidad la cuantificación enzimática de la $\beta$-Glucosidasa ácida (tanto en leucocitos como en sangre seca) solo se realiza en algunos laboratorios especializados ubicados en Bogotá, Medellín y Bucaramanga, por lo que las muestras deben ser remitidas a alguna de estas ciudades para su procesamiento.

Para la medición de la actividad enzimática en leucocitos se debe transportar sangre fresca con anticoagulante, la cual se debe mantener refrigerada durante el tras lado. Además, se debe cumplir con un tiempo límite de 24 horas entre la toma de la muestra y su análisis, para evitar que se presente la lisis de leucocitos, la cual puede alterar los resultados de la prueba. Por otro lado, una muestra tomada en papel de filtro puede ser transportada en una bolsa plástica sellada a temperatura ambiente hasta por 9 días, sin que se altere la medición de la actividad enzimática(9). Teniendo en cuenta lo anterior, el objetivo del presente estudio fue evaluar la validez de la medición de la actividad de la $\beta$-Glucosidasa ácida en sangre seca en papel de filtro, comparada con el estándar de oro (medición de la actividad enzimática en leucocitos frescos), para el diagnóstico de la enfermedad de Gaucher en pacientes con sospecha clínica de la misma, a través de una revisión sistemática de la literatura.

\section{Metodología}

El equipo investigador construyó una pregunta PICO (Tabla 1), la cual se validó con expertos del área quienes la consideraron pertinente y se publicó en la página web del Instituto de Evaluación Tecnológica en Salud (IETS), para consulta con la población general. En este proceso no se recibieron comentarios por lo que se mantuvo la pregunta PICO sin cambios.
Se diseñó una estrategia de búsqueda genérica con base en los términos clave (Gaucher Disease y Dried Blood Spot Analysis). La estrategia de búsqueda estuvo compuesta por vocabulario controlado explotado (MeSH y DeCS) y por lenguaje libre, considerando sinónimos, abreviaturas, acrónimos, variaciones ortográficas y plurales. La sintaxis se complementó con identificadores de campo, truncadores, operadores de proximidad y operadores booleanos, la búsqueda se realizó en las bases de datos MEDLINE (PubMed), Cochrane Database of systematic reviews (Wiley), Database of Abstracts of reviews of effects (Wiley) y biblioteca Virtual en Salud (LILACS). La estrategia de búsqueda se realizó de acuerdo con los estándares de calidad internacional establecidos por la Colaboración Cochrane (10).

A los resultados de las búsquedas se les aplicaron los criterios de inclusión: 1) realizar la medición de la actividad de la $\beta$-Glucosidasa ácida en leucocitos en todos los participantes, 2) realizar la medición de la actividad de la $\beta$ Glucosidasa ácida en sangre seca en todos los participantes y 3) aplicar la prueba en población con sospecha clínica de la EG; y los criterios de exclusión: 1) Artículos en idioma diferente al inglés o español y 2) artículos anteriores al 2011. $\mathrm{La}$ anterior tamización fue realizada por dos revisores de manera independiente, sin que se encontraran diferencias entre ellos, por lo que se procedió a evaluar la calidad de los artículos finales mediante la herramienta QUADAS-2 $(11,12)$

La información básica de los estudios seleccionados fue extraída por dos revisores de manera independiente basándose en un formato estándar diseñado en Excel ${ }^{\circledR}$. En caso de identificar múltiples publicaciones de un mismo estudio, o que incluyeran la misma población, se seleccionaron los datos del reporte con la fecha de publicación más reciente.

\section{Resultados}

Mediante la Búsqueda sistemática se obtuvieron 75 artículos y una vez se retiraron las referencias duplicadas quedaron 47. A estos últimos se les revisó el resumen y se seleccionaron para evaluar en texto completo los artículos de: Uribe (5), Goldim (13), Chavez (14) y Stroppiano (15).

Tabla 1. Pregunta PICO

\begin{tabular}{ll}
\hline P & Pacientes con sospecha clínica de Enfermedad de Gaucher \\
\hline I & Medición de la actividad de la $\beta$-Glucosidasa ácida en sangre "seca" sobre papel de filtro \\
C & Medición de la actividad de la $\beta$-Glucosidasa ácida en leucocitos \\
O & Presencia o ausencia de la enfermedad de Gaucher \\
\hline
\end{tabular}

Fuente: Elaboración de los autores. 
Una vez revisado el texto completo, los artículos de Uribe, Goldim y Chavez fueron excluidos por no usar el patrón de referencia diagnóstica (medición de la actividad enzimática de la $\beta$-Glucosidasa ácida en leucocitos) y no incluir los resultados negativos de la prueba índice (medición de la actividad enzimática de la $\beta$-Glucosidasa ácida en sangre seca) (Figura 1).

El artículo de Stroppiano y colaboradores(15) fue el único que cumplió los criterios de inclusión y exclusión, se trata de un estudio de casos y controles realizado en Italia en el año 2014 (Fuente: Elaboración de los autores. Tabla 2), la evaluación de la calidad metodológica según el instrumento QUADAS-2 fue de 16/18 y se consideró que no presentaba riesgo de sesgo en ninguno de los cuatro dominios. Dentro de las limitaciones de este estudio se encuentra el tamaño de muestra pequeño y el diseño de casos y controles; estas dos limitaciones se explican por la baja prevalencia de la EG.

En el artículo de Stroppiano y colaboradores se valoró la sensibilidad, la especificidad, el valor predictivo positivo y el valor predictivo negativo de la medición de la actividad enzimática de la $\beta$-Glucosidasa ácida en sangre seca al compararla con el estándar de oro (medición de la actividad enzimática de la $\beta$-Glucosidasa ácida en leucocitos),

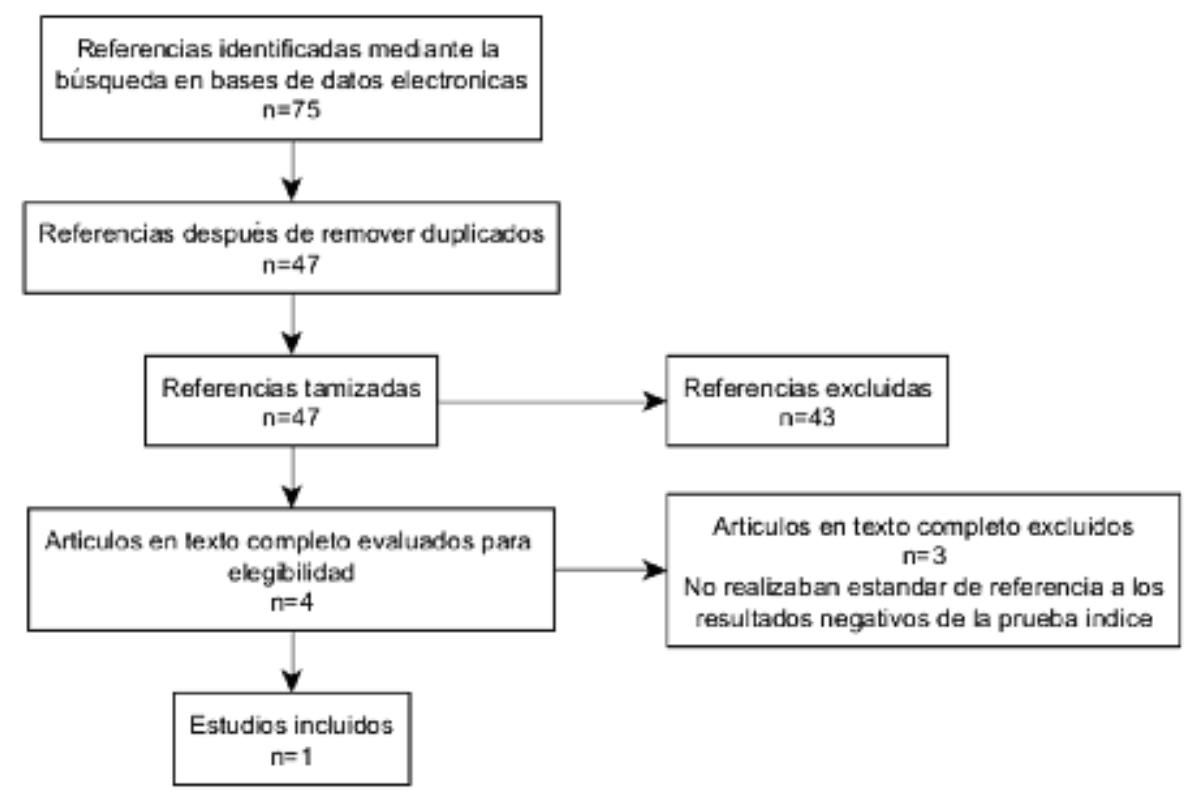

Figura 1. Diagrama de Flujo de la búsqueda, tamización y selección de la evidencia

Tabla 2. Características del estudio de Stroppiano y colaboradores

\begin{tabular}{ll}
\hline Diseño & Casos y controles \\
\hline Población & Asistentes al centro de consejería genética en Génova, Italia \\
\hline Lugar & Génova, Italia \\
\hline Comparaciones & $\beta$-Glucosidasa ácida en sangre seca Vs $\beta$-Glucosidasa ácida en leucocitos \\
\hline $\begin{array}{l}\text { Interpretación de } \\
\text { las pruebas }\end{array}$ & $\begin{array}{l}\text { Paciente sano }>2.75 \\
\text { Gaucher }(+)=<2.75\end{array}$ \\
\hline Desenlaces & Sensibilidad, especificidad, valor predictivo positivo, valor predictivo negativo \\
\hline $\begin{array}{l}\text { Tamaño de } \\
\text { muestra }\end{array}$ & 66 pacientes \\
\hline $\begin{array}{l}\text { Fuentes de } \\
\text { financiación }\end{array}$ & Genzyme (Sanofi) y el ministerio de salud de Italia \\
\hline Conclusiones & $\begin{array}{l}\text { Se debe realizar prueba en leucocitos a pesar de prueba negativa en sangre } \\
\text { seca para el diagnóstico de la enfermedad de Gaucher. }\end{array}$
\end{tabular}

Fuente: Elaboración de los autores, con datos del estudio de Stroppiano y colaboradores (15). 
Tabla 3. Rendimiento diagnóstico de la actividad enzimática de la glucocerebrosidasa en sangre seca comparada con la actividad enzimática de la glucocerebrosidasa en leucocitos.

\begin{tabular}{lcccc}
\hline Valor estimado & \multicolumn{2}{c}{ Punto de corte convencional } & \multicolumn{2}{c}{ Punto de corte ajustado } \\
\cline { 2 - 5 } & $\mathbf{0 - 2 . 7 5}$ & $\mathrm{IC} 95 \%{ }^{*}$ & $\mathbf{0 - 4 . 4}$ & $\mathrm{IC} 95 \%$ \\
\hline Sensibilidad & $82.3 \%$ & $64.0 \%-100.0 \%$ & $88.2 \%$ & $72.9-100.0 \%$ \\
\hline Especificidad & $94.0 \%$ & $91.0 \%-96.0 \%$ & $88.5 \%$ & $85.5-91.5 \%$ \\
\hline Valor predictivo positivo & $34.0 \%$ & - & $23.4 \%$ & - \\
\hline Valor predictivo negativo & $99.2 \%$ & - & $99.5 \%$ & - \\
\hline
\end{tabular}

Fuente: Modificada de Stroppiano y colaboradores (15).

teniendo en cuenta el punto de corte tradicional de $2.75 \mathrm{y}$ un nuevo punto de corte ajustado de 4.40 (Tabla 3).

\section{Discusión}

El diagnóstico de la enfermedad de Gaucher tipos I, II y III se realiza a través de la medición de la actividad enzimática de la glucocerebrosidasa en sangre seca o leucocitos. La medición de la actividad enzimática de la glucocerebrosidasa en sangre seca no tiene una capacidad diagnóstica importante, pero por su sensibilidad y la facilidad en la toma de la muestra puede utilizarse para procesos de tamización. Por lo anterior, la medición de la actividad enzimática de la $\beta$ Glucosidasa ácida en sangre seca se debe continuar utilizando como el primer eslabón de una evaluación diagnóstica serial, acompañada con medición en leucocitos.

El estudio considerado tiene algunas limitaciones metodológicas que pueden afectar la confiabilidad de las estimaciones. El diseño de casos y controles solo permite la estimación no sesgada de los verdaderos positivos y negativos, disminuyendo la predictibilidad de los estimadores de capacidad diagnóstica del test en evaluación.

Aunque la búsqueda se realizó sin límites de población en la evaluación se incluyó un artículo con población italiana, y aunque se considera que estos datos pueden extrapolarse a otras poblaciones, los resultados deben interpretarse con precaución debido a que una variación especifica del gen en alguna población podría presentar una medición diferencial entre los leucocitos frescos y la muestra de sangre seca.

No se identificaron estudios metodológicamente adecuados sobre validez de tecnologías diagnósticas para EG realizados en Colombia. El resultado disponible no contribuye con la identificación de una superioridad de la prueba diagnóstica índice comparada con la medición de la actividad enzimática de la glucocerebrosidasa en leucocitos. Por consenso con los expertos consultados, en esta evaluación de validez diagnóstica no se incluyó la evaluación de otras tecnologías usadas en el proceso diagnóstico de la EG, como son las secuenciaciones moleculares del gen GBA 1 que codifica la $\beta$-glucosidasa ácida y las mediciones iniciales de biomarcadores. Por lo tanto, la evaluación y definición del papel de estas tecnologías en el proceso de diagnóstico y manejo de EG debe ser abordado en futuros estudios sobre el tema.

\section{Conclusiones}

La medición enzimática de la $\beta$-glucosidasa ácida en sangre seca es una excelente prueba para diagnóstico inicial de EG por su buen desempeño operativo sumado a su facilidad en la toma y transporte de la muestra. No es una prueba concluyente, ya que tanto los resultados positivos como los negativos deben confirmarse a través de la medición de la actividad enzimática de la $\beta$-glucosidasa ácida en leucocitos. La evidencia presentada apoya la elección de la prueba de actividad enzimática de la $\beta$-glucosidasa ácida en leucocitos como la opción indicada para el tamizaje inicial de los pacientes sospechosos de EG.

\section{Conflicto de intereses}

Los autores declaran no tener conflictos de interés.

\section{Financiación}

La presente investigación fue financiada por el IETS.

\section{Referencias}

1. Deegan P, Hughes D, Cox T. UK National Guideline for Adult Gaucher Disease. 2005.

2. Manger B, Menge E, Schaefer R, Haase C, Seidel J, Michels H. Gaucher disease, Fabry disease and mucopolysaccharidosis type I--how can the rheumatologist recognise these patients?. Z Rheumatol. 2006; 65(1):34-43. 
3. Franco-Ornelas S. Mexican consensus on Gaucher's disease. Rev Med Inst Mex Seguro Soc. 2010; 48(2):167-86.

4. Mehta A. Epidemiology and natural history of Gaucher's disease. Eur J Intern Med. 2006; 17 Suppl:S2-5.

5. Uribe A, Giugliani R. Selective screening for lysosomal storage diseases with dried blood spots collected on filter paper in 4,700 high-risk colombian subjects. JIMD Rep. 2013; 11:107-16.

6. Sidransky E. Gaucher disease: complexity in a "simple" disorder. Mol Genet Metab. 2004; 83(1-2):6-15.

7. Fundación Española de Enfermedades Lisosomales. Actualización en Enfermedad de Gaucher [Internet]. 2008 [cited 2014 Aug 8]. Disponible en: http://www.fundacionlisosomales.es/wpcontent/uploads/2009/07/MEMORIA-FEEL-

8. Bodamer OA, Hung C. Laboratory and genetic evaluation of Gaucher disease. Wien Med Wochenschr. 2010; 160(23-24):600-4.

9. Elbin CS, Olivova P, Marashio CA, Cooper SK, Cullen E, Keutzer JM, et al. The effect of preparation, storage and shipping of dried blood spots on the activity of five lysosomal enzymes. Clin Chim Acta. 2011; 412(1314):1207-12.

10. The Cochrane Collaboration. Handbook for DTA Reviews [Internet]. 2013 [cited 2014 Sep 10]. Disponible en: http://srdta.cochrane.org/handbook-dta-reviews

11. Whiting PF, Rutjes AWS, Westwood ME, Mallett S, Deeks JJ, Reitsma JB, et al. QUADAS-2: a revised tool for the quality assessment of diagnostic accuracy studies. Ann Intern Med. 2011; 155(8):529-36.

12. Whiting $P$, Rutjes AWS, Reitsma JB, Bossuyt PMM, Kleijnen J. The development of QUADAS: a tool for the quality assessment of studies of diagnostic accuracy included in systematic reviews. BMC Med Res Methodol. 2003; 3(1)3:25.

13. Goldim MP de S, Garcia C da S, de Castilhos CD, Daitx VV, Mezzalira J, Breier AC, et al. Screening of high-risk Gaucher disease patients in Brazil using miniaturized dried blood spots and leukocyte techniques. Gene. 2012; 508(2):197-8.

14. Chaves RG, Coelho JC, Michelin-Tirelli K, Maurício TF, de Freitas Maia Chaves E, de Almeida PC, et al. Successful screening for Gaucher disease in a highprevalence population in tabuleiro do Norte (northeastern Brazil): a cross-sectional study. JIMD Rep. 2011; 1:73-8.

15. Stroppiano M, Calevo MG, Corsolini F, Cassanello M, Cassinerio E, Lanza F, et al. Validity of $\beta$-d-glucosidase activity measured in dried blood samples for detection of potential Gaucher disease patients. Clin Biochem. 2014; 47(13-14):1293-96. 